\title{
Squamous cell carcinoma of the temporal bone in 30 patients: Difference in presentation and treatment in de novo disease I vs radiation associated disease
}

\section{1 | INTRODUCTION}

Squamous cell carcinoma temporal bone (SCCTB) is a rare malignancy with incidence of around 1 to 1.2 case per million populations per year. Risk factors included chronic otitis media and chronic irritation of the skin and mucosa of the external and middle ear. ${ }^{1} \mathrm{~A}$ rare but important aetiology is radiation to the region for treatment of head and neck malignancies. Radiation treatment for nasopharyngeal carcinoma (NPC) appears an important association of post-irradiated SCC of temporal bone in South-East Asia as demonstrated by previous reported cohorts from Taiwan and Singapore. ${ }^{2-4}$

This study investigates and compares the presentation, treatment and prognosis of de novo SCCTB ( $\mathrm{nnSCC}$ ) and post-irradiated SCCTB (piSCC). We investigate whether prior irradiation to the region would limit on treatment options and leading to difference in survival and local disease control of piSCC compared to dnSCC.

\section{METHODS}

We reviewed our department's database and retrieved all temporal bone squamous cell carcinoma treated from June 1995 to June 2016. Only patients with complete clinical data were included. The study was approved by our hospital's ethics committee.

All patients have histological diagnosis of SCC of the external auditory canal or mastoid. SCC of the auricle and pinna was excluded. $36.7 \%$ patients had MRI scan in addition to CT scan performed for tumour staging. In our institute, all the patients with NPC were followed up lifelong by the otorhinolaryngologist.

We analysed the clinical characteristics and treatment outcome and further divided the patients into de novo group ( $\mathrm{dnSCC}$ ) with no prior radiation to the head and neck region and the piSCC, who all had received radiation treatment. Post-irradiation malignancy is defined as development of a new malignancy with different histology within the field of previous radiation treatment diagnosed after a latent period of 5 years, and which is histologically different from the initial malignancy. ${ }^{5}$ Modified Pittsburgh Classification ${ }^{6}$ was used to stage the SCC temporal bone. ${ }^{6}$ Comparisons were made on the stage at presentation, disease-free survival (DFS) and overall survival (OS).

All data were collected retrospectively. SPSS version 23 was used for analysis. Kaplan-Meier plot and log rank test are used for survival analysis. Mann-Whitney $U$ test for continuous variables and the Fisher's exact test for categorical variables were used for analysis. A $P$ value of less than .05 was considered as significant.

\section{RESULTS}

\section{1 | Patient demographics}

From June 1995 to June 2016, 30 cases of temporal bone SCC were treated in our institution, of which $16(53.3 \%)$ were piSCC and 14 $(46.7 \%)$ were dnSCC. All the patients in piSCC group received radiation treatment for NPC.

There was no difference in baseline characteristics (Table 1A) Mean age of SCC presentation was 64.8 years old. In the piSCC group, the mean age of diagnosis of the NPC was 48.3 years old Male pre-dominance was observed in both groups.

$10 \%$ of the tumour has initial negative biopsy result, including squamous papilloma, inflamed granulation tissue, cholesteatoma and inflammatory polyp. Histological diagnosis of SCC temporal bone was confirmed in later biopsy or operative specimen.

The median latency period of piSCC is 16.8 years, ranges from 5.76 to 29.1 years. It is consistent with previous reports that the median latency period of piSCC is 17-18.8 years and can be as long as three decades. ${ }^{3,4}$

In our current cohort, bilateral sequential piSCC of temporal bone occurred in one male and one female patient with NPC, the latency period is 12.6 year and 10.0 year, respectively. The first incidence of SCC temporal bone of the female patient occurred in 1992; therefore, the episode was excluded from the current cohort. The incidence of sequential contralateral piSCC of temporal bone is $13.3 \%$.

\section{2 | Stage of disease at presentation}

Majority of patients presented late at Pittsburg T3 and T4 disease, constituting $27 \%$ and $36 \%$ of the whole cohort, respectively. Postirradiated SCCTB presented significantly earlier with lower Pittsburg T-stage and overall stage. Two patients in the piSCC group presented early with Tis disease. (Table 1B).

Lymph node metastasis is uncommon at presentation, in which only two patients (7\%) had N2 disease at presentation. There was no statistical difference in the initial N Stage (Table 1B). 
TABLE $1 \quad X X X X X$

5

\begin{tabular}{lrrrr|} 
& $\begin{array}{c}\text { Whole } \\
\text { cohort }\end{array}$ & dnSCC & $\begin{array}{l}\text { Post-irradiated } \\
\text { SCCTB }\end{array}$ & P value \\
\hline Age of diagnosis (y) & & & & \\
$\quad \begin{array}{l}\text { Mean } \\
\text { Range }\end{array}$ & 64.8 & 64.0 & 65.5 & .754 \\
Gender & $46-84.5$ & $46-84.5$ & $49.3-80.2$ & \\
\hline $\begin{array}{l}\text { Male } \\
\text { Female }\end{array}$ & 23 & 11 & 13 & .63 \\
\hline $\begin{array}{l}\text { History of chronic } \\
\text { ear infection }\end{array}$ & 6 & 3 & 3 & \\
\hline
\end{tabular}

\section{3 | Treatment}

Two patients received radiotherapy alone and one patient received chemo-radiation alone. 25 cases were treated with curative intent surgery and two cases in the piSCC group were treated with palliative surgery for pain relief: $11(40.7 \%)$ radical mastoidectomies, 10 (37.0\%) lateral temporal bone resections and six (22.2\%) subtotal temporal bone resections. Resection margin was positive or close in six patients with piSCC (42.9\%) and seven patients with dnSCC (50.0\%). The commonest involved margins are the deep and/ or superior margin, occurring in $69.2 \%$ cases of involved margins.

Neck dissection was performed on the two patients who presented with lymph node metastasis and one patient requiring free flap reconstruction. Elective neck dissection was not performed in patients with NO disease. Two patients received additional parotidectomy and two patients received condylectomy for tumours extending anteriorly to the parotid or temporomandibular joint.

Adjuvant radiotherapy or chemoradiotherapy were offered to patients with T3 or T4 tumour or positive resection margins, where the decision for adjuvant treatment was made in multidisciplinary meeting involving oncologist and surgeons. Nevertheless, five of seven patients in piSCC group (71.4\%) refused adjuvant radiotherapy after discussion with oncologist due to toxicity of the additional second or third course of radiotherapy when majority had already suffered from significant complication of prior radiation for NPC. Significantly more patients (84.6\%) in dnSCC group received postoperative adjuvant treatment comparing to only $14.3 \%$ of the piSCC 6 group (Table 2).

\section{4 | Outcome}

The follow-up period ranged from five to 245 months. Estimated median OS, disease-specific survival (DSS) and DFS of the whole cohort were 45, 48 and 18 months, respectively.

Median OS of dnSCC group and piSCC group is 23 months (95\% $\mathrm{Cl}$ : 0-54.8 months) and 48 months (95\% Cl: 33.4-62.6 months), respectively (Figure 1A). Median DSS of dnSCC group and piSCC group is 43 months (95\% Cl: $43.0-56.0$ months) and 56 months (95\% Cl: $37.0-75.0$ months), respectively (Figure 1B). There was no

\section{Keypoints}

- History of nasopharyngeal carcinoma (NPC) is the most important cause of post-irradiated SCCTB (piSCC) of the temporal bone in locality where NPC is endemic, including Hong Kong.

- Bilateral sequential piSCC in NPC survivors is not uncommon. $13 \%$ of patient with piSCC in our series has bilateral disease.

- Long latency period of piSCC indicates lifelong surveillance of patients with NPC might be necessary, in which early detection can be achieved as demonstrated in our series.

- Lower T-stage and overall stage can translate to superior overall and DFS of SCC temporal bone.

- Prognosis of piSCC and dnSCC remains similar despite early detection of piSCC in our series, which may be attributed by the limitation in administration of adjuvant treatment in patients with piSCC, and therefore, achieving a negative surgical margins is seemed to offer best outcome for patients with piSCC temporal bone.

statistical difference in the OS and DSS between the dnSCC and piSCC group despite early T-stage diseases in the piSCC group.

As expected, OS and DFS are significantly better in tumour with lower T-stage. The 3-year OS of T2 or lower stage is 100\%. 3-year OS survival markedly reduced to $50 \%$ for T3 tumour and $31.2 \%$ for T4 tumour (Figure 2A). Similarly, 3-year DFS of Tis and T1 tumour achieved 100\%, and T2 tumour has achieved 75\% (Figure 2B).

\section{TABLE 2 XXXXX}

\begin{tabular}{|ccccc|}
\hline & $\begin{array}{c}\text { Whole } \\
\text { cohort }\end{array}$ & dnsCC & $\begin{array}{l}\text { Post-irradiated } \\
\text { SCCTB }\end{array}$ & P value \\
\hline T stage (\%) & & & & \\
\hline Tis & $2(7)$ & $0(0)$ & $2(12)$ & .034 \\
T1 & $4(13)$ & $1(7)$ & $3(19)$ & \\
T2 & $5(17)$ & $1(7)$ & $4(25)$ & \\
T3 & $8(27)$ & $5(36)$ & $3(19)$ & \\
T4 & $11(36)$ & $7(50)$ & $4(25)$ & \\
\hline N stage (\%) & & & \\
N0 & $28(93)$ & $12(86)$ & $16(100)$ & \\
N1 & $0(0)$ & $0(0)$ & $0(0)$ & \\
N2 & $2(7)$ & $2(14)$ & $0(0)$ & \\
\hline
\end{tabular}

Overall stage (\%)

$\begin{array}{lcll}\text { Stage 0 } & 2(7) & 0(0) & 2(12) \\ \text { Stage I } & 4(13) & 1(7) & 3(19) \\ \text { Stage II } & 5(17) & 1(7) & 4(25) \\ \text { Stage III } & 7(23) & 4(29) & 3(19) \\ \text { Stage IV } & 12(40) & 8(57) & 4(25)\end{array}$



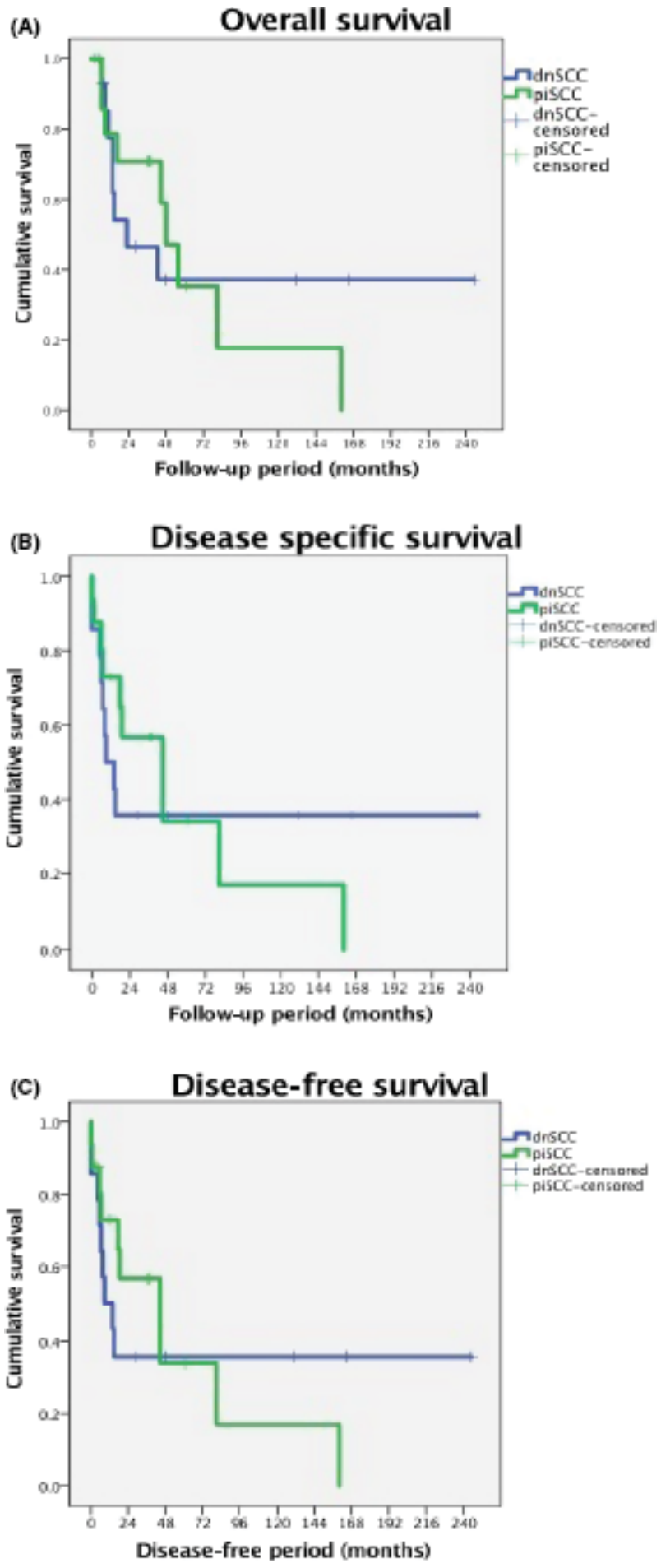

10 FIGURE 1 (A) Kaplan-Meier survival curve showing overall survival in piSCC group compared with dnSCC group. $P=.887$, log rank test; (B) Kaplan-Meier survival curve showing disease-specific survival in piSCC group compared with dnSCC group. $P=.803$, log rank test; (C) Kaplan-Meier survival curve showing disease-free survival in piSCC group compared with dnSCC group. $P=.562$, log rank test\%

None of the patients with piSCC develop distant or nodal metastasis within the follow-up period, comparing to $35.7 \%$ in the dnSCC group.
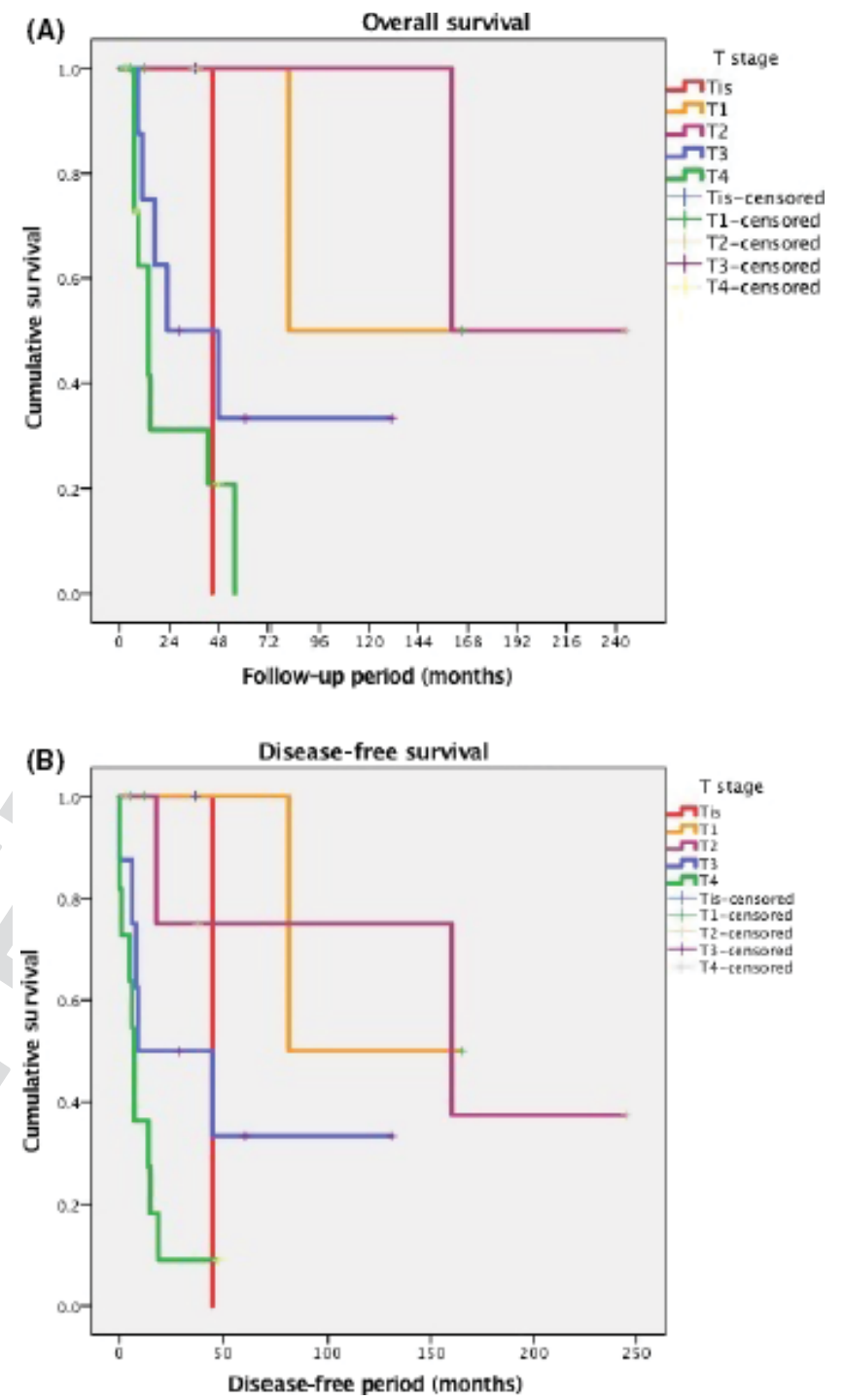

FIGURE 2 (A) Kaplan-Meier survival curve showing overall survival of SCC temporal bone categorized in T stage. $P=.023$, log rank test; (B) Kaplan-Meier survival curve showing disease-free survival of SCC temporal bone categorized in T stage. $P=.014$, log rank test

\section{4 | DISCUSSION}

Nasopharyngeal carcinoma is prevalent in Hong Kong. According to the Hong Kong Cancer Registry, yearly incidence of NPC in our locality ranges from 800-900. Prior radiation therapy for NPC is the most common cause of piSCC temporal bone in Hong Kong, constituting all cases in our series.

Due to rarity of disease, management strategies were guided by limited number of available cohort series and literature reviews. $^{7-9}$ In particular, limited information on piSCC was available in the literature - Lim et al. first reported five cases in $2000 .^{2}$ Lo et al. ${ }^{3}$ reported 10 cases in 2008, and Tay el al. ${ }^{4}$ reported eight cases in 2014. All the three cases series were NPC survivors. Most recently in 2015, Lambert et al. ${ }^{10}$ from MD Anderson Cancer Centre in US reported nine cases, which a proportion of the primary tumours were not NPC. Treatment strategies for 
example the choice of surgical approach with en bloc vs piecemeal dissection, need of elective parotidectomy, need of elective neck dissection, effectiveness of chemotherapy were much debated and remained controversial. ${ }^{7-9}$

Although adjuvant radiation is generally indicated in SCCTB of advanced stage or aggressive features, ${ }^{8}$ further irradiation to $\mathrm{piSCC}$ is limited by previous radiation exposure of the temporal bone. Patients with post-irradiated NPC tolerated further irradiation poorly. In the series by Lo et al.., ${ }^{1} 50 \%$ of the patients with piSCC received further adjuvant radiotherapy (50-60 Gy) died of radiation complication (two massive epistaxis, one temporal bone osteoradionecrosis). Therefore, surgery remains the mainstay of treatment in this group of patient, with achieving a negative surgical margins seemed to offer the best outcome for patients with PISCC of the ear. ${ }^{4}$

In the present cohort, piSCC group of patients presents with earlier disease then dnSCC group. This may be due to the regular follow up of the patients with NPC by otolaryngologist and early biopsy of suspicious lesions in the ear. On the contrary, patients with dnSCC usually have chronically discharging ears that they elect to disregard the symptoms and not seek medical care until when symptoms of more advance disease like pain and significant bleeding occur.

It would be expected that with earlier presentation, the piSCC group should enjoy a better survival. However, adjuvant radiation was usually not given to piSCC group of patients after radical surgery to avoid excessive toxicity to the surrounding tissue. The lack of adjuvant radiation in the piSCC group may explain the similar disease control and survival despite earlier presentation of the disease. As the anatomy of the temporal bone is complex with many vital structures in proximity, a true negative resection margins may not be easily achieve and adjuvant radiation may have a role in controlling the microscopic residual diseases.

Survival of piSCC is generally poor, the 3-year OS ranges from $20 \%-60 \%$. Literature data on comparison prognosis of piSCC and dnSCC remained inconsistent. Lo et al. ${ }^{3}$ in 2000 suggested inferior prognosis in patients with piSCC, whereas latest series in 2014 by Tay et al. ${ }^{4}$ suggested similar prognosis between the two groups. Optimal treatment strategy and surgical approach still remains controversial.

Role of elective neck dissection and parotidectomy is controversial. $^{7-9}$ In our series, all patients with piSCC did not receive elective neck dissection and elective parotidectomy. No patients with piSCC develop nodal or distant metastasis. Therefore, elective neck dissection and elective parotidectomy in patients with prior irradiation to the neck are not recommended.

This is the largest cohort of temporal bone SCC occurring in patient who had prior radiation to the head and neck region reported in the literature. Nevertheless, retrospective nature subjects the study to misclassification and information bias. Data collection spans over 15 years, 3-D conformal RT has emerged as the treatment of choice over the years and the choice of surgical treatment by en bloc vs piecemeal surgery was based on the discretion of surgeon. Histological assessment of margins was non-standardised and it is difficult to ascertain the surgical margins in case of piecemeal resection.

\section{CONCLUSION}

Post-irradiated SCCTB of temporal bone is rare but important complication in NPC patients with prolonged survival. Majority, if not all piSCC in our locality occur in patients with history of NPC. Long latency period of the disease indicates lifelong surveillance of patients with NPC might be necessary. It is important to be aware of bilateral sequential piSCC as it can occur in up to $13 \%$ of patients.

Early detection can be achieved in long-term survival as demonstrated in our series. High index of suspicion is required due to the inconspicuous signs and symptoms as well as possible initial falsenegative biopsy result. Although lower T-stage and overall stage can translate to superior overall and DFS, prognosis of piSCC and dnSCC remains similar. It may be attributed by limitation in administration of adjuvant treatment in patients with piSCC. Elective neck dissection and parotidectomy are not recommended in patients with prior irradiation to neck.

\section{CONFLICT OF INTEREST}

None to declare.

H.Y. Sun ${ }^{1}$
R.K. Tsang ${ }^{2}$
Division of Otolaryngology, Department of Surgery, University of Hong
Kong, Hong Kong, China
${ }^{2}$ Department of Surgery, Queen Mary Hospital, Hong Kong, China
Correspondence
R.K. Tsang, Department of Surgery, Queen Mary Hospital, Hong
Kong, China.
Email: rkytsang@hku.hk

\section{REFERENCES}

1. Kuhel WI, Hume CR, Selesnick SH. Cancer of the external auditory canal and temporal bone. Otolaryngol Clin North Am. 1996;29:827-852.

2. Lim LH, Goh YH, Chan YM, Chong VF, Low WK. Malignancy of the temporal bone and external auditory canal. Otolaryngol Head Neck Surg. 2000;122:882-886.

3. Lo WC, Ting LL, Ko JY, et al. Malignancies of the ear in irradiated patients of nasopharyngeal carcinoma. Laryngoscope. 2008;118:2151-2155.

4. Tay G, Tan HK, Thiagarajan A, Soo KC, Iyer NG. Squamous cell carcinoma of the ear arising in patients after radiotherapy for nasopharyngeal carcinoma. Eur Arch Otorhinolaryngol. 2014;271: 149-156.

5. Cahan WG, Woodard HQ, et al. Sarcoma arising in irradiated bone; report of 11 cases. Cancer. 1948;1:3-29.

6. Moody SA, Hirsch BE, Myers EN. Squamous cell carcinoma of the external auditory canal: an evaluation of a staging system. Am J Otol. 2000;21:582-588.

7. Zanoletti ????, Lovato ????, Stritoni ????, Martini ????, Mazzoni ????, Marioni ????. A critical look at persistent problems in the diagnosis, staging and treatment of temporal bone carcinoma. Cancer Treat Rev. 2015;41:821-826.

8. Lionello ????, Stritoni ????, Facciolo ????, et al. Temporal bone carcinoma. Current diagnostic, therapeutic, and prognostic concepts. J Surg Oncol. 2014;110:383-392. 
9. Prasad S, D'Orazio F, Medina M, Bacciu A, Sanna M. State of the art in temporal bone malignancies. Curr Opin Otolaryngol Head Neck Surg. 2014;22:154-165.
10. Lambert EM, Garden AS, DeMonte F, Roberts DB, Gidley PW. Radiation- associated malignancies of the ear canal and temporal bone. Laryngoscope. 2015;125:1198-1204. 